\title{
Problem in Plane Geometry
}

By M. Edouard Collignon, Inspecteur général des Ponts et Chausées en retraite, Examinateur honoraire a l'Ecole polytechnique, Paris.

(Read 11th December 1908.)

\section{Chapter I}

The principal problem which we have in view may be stated as follows :

T'o construct a triangle ABC (Frig. 1) in which are given in magnitude only, the height $h=\mathrm{AO}$ from the vertex $\mathrm{A}$, the median $m=\mathrm{BI}$ from the vertex $\mathrm{B}$, and the bisector $f^{\circ}=\mathrm{CD}$ from the vertex $C$.
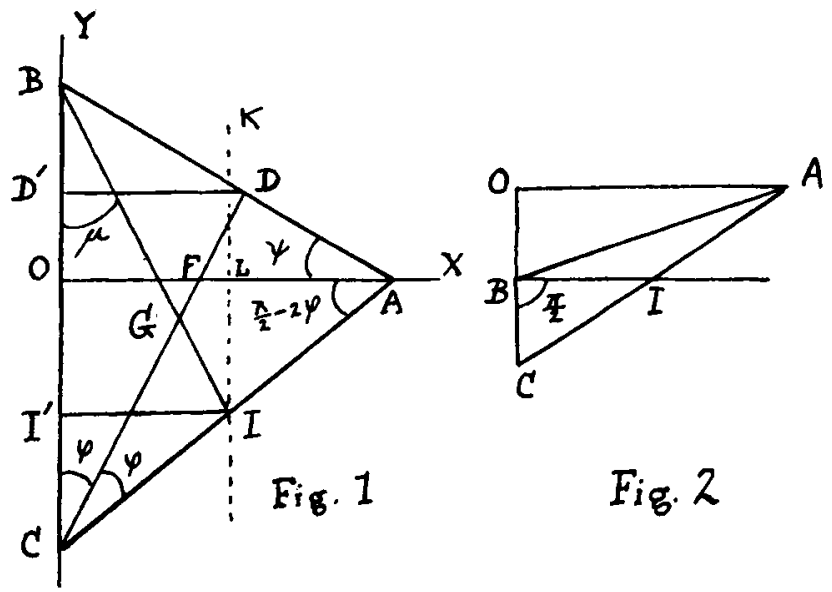

The position of one of them can always be chosen arbitrarily, e.g., the height. When once this choice is made, we take as our rectangular axes,

$1^{\circ}$ the direction OA already given to the height $h$, as $x$-axis $O X$;

$2^{\circ}$ the direction of the side $\mathrm{CB}$, perpendicular to $\mathrm{OX}$ at $\mathrm{O}$, as $y$-axis $\mathrm{OY}$. 
We proceed to discuss in succession the conditions of the problem relative to the median and the bisector, which are the only data remaining to be fixed.

\section{Condition relative to the Median}

Let I be the mid point of $\mathrm{AC}$; then $\mathrm{BI}$ will be the median whose length must equal $m$. If we rotate the side $C A$ about the fixed point $A$, the point $I$ will trace out a straight line IK parallel to $O Y$ at a distance $I^{\prime} I=\frac{h}{2}$ from it. Let $\mu$ be the angle IBC which the median makes with the side BC. $\mu$ is determined by the construction for

$$
\sin \mu=\mathrm{I}^{\prime} \mathrm{T} / \mathrm{BI}=h / 2 m \text {. }
$$

If the triangle $\mathrm{ABC}$ is possible with the given data, $h \leqq 2 m$. If in particular $h=m, \mu=30^{\circ}$. The solution of (1) gives two supplementary angles for $\mu$, which are both equal to $\frac{\pi}{2}$ when $h=2 m$. In this case the triangle $\mathrm{ABC}$ has its vertex $\mathrm{B}$ at the mid point of $\mathrm{OC}$ (Fig. 2). Hence the median $\mathrm{BI}$ is parallel to $\mathrm{OA}$, and the angle IBC is equal to $\frac{\pi}{2}$.

The angle $\mu$ is greater than $\frac{\pi}{2}$ if the vertex $B$ is lower than the mid point of $\mathrm{OC}$.

From (1) we have $\cos \mu= \pm \frac{1}{2 m} \sqrt{ }\left(4 m^{2}-h^{2}\right)$. The product $m \cos \mu$ is then $\pm \sqrt{ }\left(4 m^{2}-h^{2}\right)$, which is equal to $\cot \mu$ since $m$ is $h / 2 \sin \mu$. The factor $\cot \mu$ is positive or negative according as $\mu$ is acute or obtuse. The sign can be chosen to suit the particular conditions of the problem.

\section{Choice of unknown Variable}

Draw the bisector $\mathrm{CD}$ of the angle $\mathrm{C}$, and let

$$
\angle \mathrm{ACD}=\angle \mathrm{DCB}=\phi \quad \text { (Fig. 1.) }
$$

If this angle were known the point $\mathrm{C}$ could be obtained by making at $\mathrm{A}$ the angle $\mathrm{OAC}=\frac{\pi}{2}-2 \phi$, complement to $\mathrm{OCA}=2 \phi$. 
The angle $\phi$, half of an angle of a triangle, is necessarily acute. Then having drawn the side AC, if with the middle point $\mathrm{I}$ as centre, we draw a circle of radius $m$ cutting $O Y$ in $B$, the triangle will be completely determined.

From Figure 1 we have the following relations:

$$
\begin{gathered}
\mathrm{AC}=b=h / \sin 2 \phi ; \mathrm{CB}=a=m \cos \mu+\frac{1}{2} h \cot 2 \phi=\frac{h}{2}(\cot \mu+\cot 2 \phi) ; \\
\mathrm{OB}=m \cos \mu-\frac{h}{2} \cot 2 \phi=\frac{h}{2}(\cot \mu-\cot 2 \phi) .
\end{gathered}
$$

If we call $\psi$ the angle $\mathrm{BAO}$, which added to $\mathrm{OAC}=\frac{\pi}{2}-2 \phi$, gives the angle $A$ of the triangle, we have

$$
\begin{gathered}
\tan \psi=\mathrm{OB} / h=\frac{1}{2}(\cot \mu-\cot 2 \phi), \mathrm{A}=\frac{\pi}{2}-2 \phi+\psi, \text { and } \\
\mathrm{AB}=c=h / \sin \psi .
\end{gathered}
$$

The area $\mathrm{S}$ of the triangle $\mathrm{ABC}$ is $\frac{1}{2} a h=\frac{1}{2} h^{2}(\cot \mu+\cot 2 \phi)$. The angle $\phi$ is thus the key of the solution.

If we draw from the point $D$, the foot of the bisector $C D$, a perpendicular $\mathrm{DD}^{\prime}$ on $\mathrm{OY}$, we get as co-ordinates of $\mathrm{D}$ with respect to $\mathrm{OX}, \mathrm{OY}$

$$
\begin{aligned}
x=\mathrm{D}^{\prime} \mathrm{D}= & f \sin \phi, y=\mathrm{OD}^{\prime}=f \cos \phi-h \cot 2 \phi . \\
& \text { Auxiliary curves }
\end{aligned}
$$

\section{$1^{\circ}$ The envelope of the bisector $C D$}

We wish to find the envelope of the bisector CD when the side $\mathrm{AC}$ is turned about the fixed point $\mathrm{A}$ by varying the angle $\phi$.

Iet $F$ be the point of intersection of $C D$ and $O X$ (Fig. 1). The angle DFA is the complement of $\phi$, and the direction coefficient of $\mathrm{CD}$ is $\tan \mathrm{DFA}=\cot \phi$. The ordinate $\mathrm{OC}$ of $\mathrm{C}$ is $-h \cot 2 \phi$, and hence the equation of $\mathrm{CD}$ is

$$
y=x \cot \phi-h \cot 2 \phi \text {. }
$$

To get the equation of the envelope, differentiate $(a)$ with respect to $\phi$. We get

$$
0=\frac{x}{\sin ^{2} \phi}-\frac{2 h}{\sin ^{2} 2 \phi} \text {. }
$$


This equation gives the abscissa $x$ of the point where the moving line touches its envelope. Thus

(c)

$$
x=h / 2 \cos ^{2} \phi \text {; }
$$

and from $(a)$ the corresponding ordinate is found to be

$$
y=\frac{h \cot \phi}{2 \cos ^{2} \phi}-h \cot 2 \phi=h \tan \phi .
$$

Eliminating $\phi$ from (c) by means of $(d)$, we get the equation of the envelope :

(e)

$$
y^{2}=2 h x-h^{2} .
$$

This represents a parabola $\mathrm{L}^{\prime} \mathrm{LML}$ (Fig. 3) with $\mathrm{A}$ as focus, $O A$ as axis, $O Y$ as directrix, and $L$, the intersection of IK with OA (Fig. 1), as vertex. The subnormal is the constant $\mathrm{OA}=\boldsymbol{h}$.

It is easy to verify from the figure the above results.

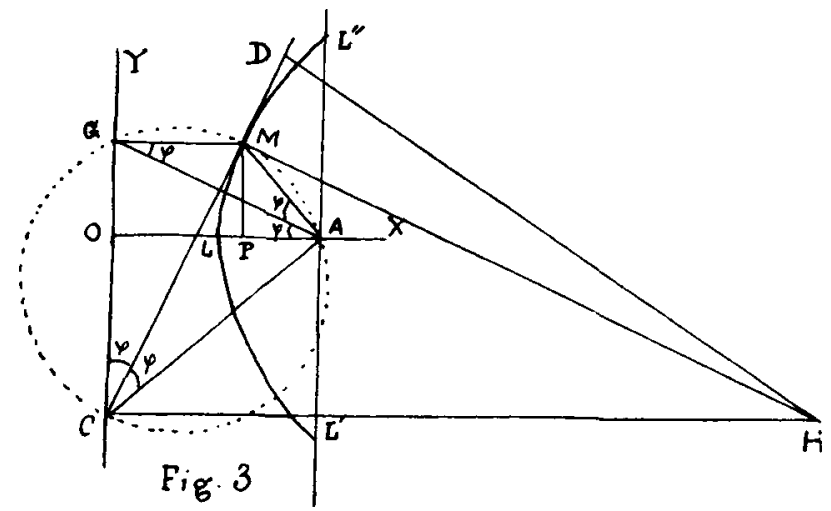

The parabola is the locus of the point M, whose distance from $A$ is equal to its distance from $O Y$, and the tangent $M C$ is the bisector of the angle GMA. Therefore $\mathrm{CA}=\mathrm{CG}$, and the tangent also bisects ACG. The quadrilateral AMGC is cyclic and $\phi=\mathrm{GCM}=\mathrm{MCA}=\mathrm{MAG}=\mathrm{MGA}=\mathrm{GAO}$.

Hence the ordinate $\mathrm{PM}=\mathrm{OG}=h \tan \phi$ as in $(d)$, and

$\mathrm{OP}=\mathrm{GM}=\mathrm{GA} / 2 \cos \dot{\phi}=\mathrm{OA} / 2 \cos ^{2} \phi=h / 2 \cos ^{2} \phi$ as in $(c)$.

The Minimum of the intercept of the tangent to the parabola between the directrix and the curve

Let $(x, y)$ be the point $M$ at which we draw the tangent MC to the parabola, given by the equation $y^{2}=2 h x-k^{2}$. 
The equation of the tangent is

$$
y^{\prime}-y=\frac{h}{y}\left(x^{\prime}-x\right) \text {. }
$$

The difference $y^{\prime}-y$, in absolute value, represents the projection GC of MC on OY when we put $x^{\prime}=0$.

$$
\therefore \mathrm{MC}^{2}=\mathrm{OP}^{2}+\left(y^{\prime}-y\right)^{2}=x^{2}\left(1+\frac{h^{2}}{y^{2}}\right)=x^{2}+\frac{h^{2} x^{2}}{2 h x-h^{2}}=\frac{x^{3}}{x-\frac{h}{2}}
$$

Equating the derivative of this to zero, we have

$$
\frac{2 x^{3}-\frac{3}{2} h x^{2}}{\left(x-\frac{h}{2}\right)^{2}}=0 \text {. }
$$

As $x$ cannot be equal to zero we get

$$
2 x=\frac{3}{2} h \text { or } x=\frac{3}{4} \mathrm{OA} \text {. }
$$

The corresponding ordinate is $\sqrt{ }\left(\frac{3}{2} h^{2}-h^{2}\right)=\frac{h}{\sqrt{ } 2}$.

To this point corresponds an angle $\phi$ given by the equation

$$
\tan \phi=\frac{y}{h}=\frac{1}{\sqrt{ } 2}, \text { from which } \phi=39^{\circ} 16^{\prime} .
$$

The corresponding length of the tangent is

$$
\mathrm{MC}=x \sqrt{ }\left(1+\frac{h^{2}}{y^{2}}\right)=\frac{3 \sqrt{ } 3}{4} h .
$$

\section{$2^{\circ}$. Geometrical locus of $D$ the foot of the bisector $C D$}

The co-ordinates of the point $\mathrm{D}$ are given by the equations :-

$$
x=f \sin \phi, \quad y=f \cos \phi-h \cot 2 \phi .
$$

From these we must eliminate the parameter $\phi$. The equation of the locus of $D$ is

$$
y=\sqrt{ }\left(f^{2}-x^{2}\right)-\frac{h\left(f^{2}-2 x^{2}\right)}{2 x \sqrt{ }\left(f^{2}-x^{2}\right)} .
$$


The square root $\sqrt{ }\left(f^{2}-x^{2}\right)$ disappears on squaring, and we thus get an equation of the sixth degree.

For the discussion of the curve it seems preferable to keep the equation in the form (3), or even in the form of the two equations (2). When $x=0, y=-\infty$ and when $x=f, y=+\infty$.

The curve is contained between the two parallels $x=0, x=f$, or more generally between the parallels $x=-f, x=+f$ if negative abscissae are allowed. The curve $m n p$ (Fig. 4) approaches its asymptotes at the positive infinity for $x=f$ and at the negative for $x=0$. Between the two points, the infinite branch has a point of inflexion $I$, whose precise position is obtained by equating to zero the second derivative $\frac{d^{2} y}{d x^{2}}$. The formation of this derivative is verylaborious, and gives for the determination of the abscissa $x$ a very complex equation.

Reduced to its first term the equation (3) becomes $y=\sqrt{ }\left(f^{\prime 2}-x^{2}\right)$, which represents a circle with centre $O$ and radius $f$.

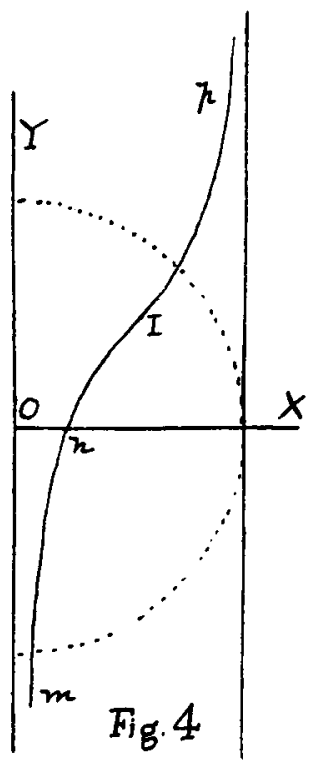

The neglected term $-\frac{h\left(f^{2}-2 x^{2}\right)}{2 x \sqrt{ }\left(f^{2}-x^{2}\right)}$ is the variation which must be made in the ordinate of the circle to obtain the ordinate of the curve. This variation is infinite in absolute value for $x=0$ and $x=f$, i.e., at the points where the circle meets the axes. It is zero for $x= \pm f / \sqrt{ } 2, i e$, at the intersections of the circle with the bisectors of the angles between the axes. The curve cuts the axis $\mathrm{OX}$ at points given by the equation

$$
2 x\left(f^{2}-x^{2}\right)=h\left(f^{2}-2 x^{2}\right),
$$

which, arranged in powers of $x$, gives

$$
\mathrm{P} \equiv x^{3}-h x^{2}-f^{2} x+\frac{h f^{2}}{2}=0 .
$$


$\mathrm{P}$ becomes $\frac{h f^{2}}{2}$ for $x=0$ and $-\frac{h^{3}}{8}$ for $x=\frac{h}{2}$ which shows that there is a root between $x=0$ and $x=\frac{h}{2}$.

From equations (2) we obtain

$$
\frac{d y}{d x}=\frac{h}{2 \int \sin ^{2} \phi \cos ^{3} \phi}-\tan \phi .
$$

The construction for the tangent, or rather for the normal to the locus of $\mathrm{D}$ is found from the consideration of an infinitely small displacement of the moving line CD of constant length $f$. The point $\mathrm{C}$ moves along $\mathrm{OY}$, and the line $\mathrm{CD}$ itself is tangent at $\mathrm{M}$ to the parabola (Fig. 3). Thus the instantaneous centre of rotation of the line is the point of intersection of the normals $\mathrm{CH}$ and MH to the trajectories of the points $\mathrm{C}$ and $\mathrm{M}$, and the straight line $\mathrm{HD}$ is the normal to the trajectory of $\mathrm{D}$. There is on the parabola a point $M$ for which $\mathbf{C M}=f$; at this point the two curves, the parabola and the locus of $\mathrm{D}$, meet. The construction for the normal shows that they touch at this common point, because the normals $\mathrm{HD}$ and $\mathrm{HM}$ are coincident. This result is evident since the point $D$ on the tangent $\mathrm{CM}$ must always remain outside the parabola. To find where the tangent $\mathbf{C M}$ has the length $f$, we equate to $f$ the length of the tangent and obtain

or

$$
\begin{gathered}
x \sqrt{ }\left(1+\frac{h^{2}}{y^{2}}\right)=f \\
y= \pm h x / \sqrt{\left(f^{2}-x^{2}\right)} .
\end{gathered}
$$

The point also belongs to the curve (3) the locus of $\mathrm{D}$ since it corresponds to the equation $\mathrm{CM}=\mathrm{CD}$. The abscissa of the point is therefore given by the equation

$$
\frac{h x}{\sqrt{\left(f^{2}-x^{2}\right)}}=\sqrt{ }\left(f^{2}-x^{2}\right)-\frac{h\left(f^{2}-2 x^{2}\right)}{2 x \sqrt{ }\left(f^{2}-x^{2}\right)}
$$

or, on simplification,

$$
x^{3}-f^{2} x+\frac{1}{2} h f^{2}=0 .
$$

After the substitution $x=f x^{\prime}$ we have to solve the cubic equation

$$
x^{3}-x^{\prime}+h / 2 f=0
$$


Note on the locus of $D$ form

Consider again the equation (3) which may be written in the

or

$$
\begin{gathered}
y=\sqrt{ }\left(f^{2}-x^{2}\right)\left[1-\frac{h\left(f^{2}-2 x^{2}\right)}{2 x\left(f^{2}-x^{2}\right)}\right] \\
\frac{y}{\sqrt{ }\left(f^{2}-x^{2}\right)}=1-\frac{h}{2 x}+\frac{h}{4(f-x)}-\frac{h}{4(f+x)} .
\end{gathered}
$$

The terms on the right can be constructed geometrically by choosing a unit of length suitable to the ordinates of the figure. Take the three equilateral hyperbolae
(i) $z_{1}=-\frac{h}{2 x}$;
(ii) $z_{2}=\frac{h}{4(f-x)}$;
(iii) $z_{3}=-\frac{h}{4(f+x)}$

The ordinates of the three curves are of unit length when $x=\frac{h}{2}, f+x=\frac{h}{4}$ and $f-x=\frac{h}{4}$ respectively.

The first hyperbola $\left(z_{1}, x\right)$ has for asymptotes the axes $O X$ and $O Y$; the second $\left(z_{2}, x\right)$ has as asymptotes the axis $O X$ and the line $x=f$; and the third $\left(z_{3}, x\right)$ has as asymptotes $\mathrm{OX}$ and $x=-f$.

The two hyperbolae (ii) and (iii) are symmetrically placed with respect to the origin $O$ (Fig. 5). The parameter of (1) is, irrespective of sign, the double of those of (ii) and (iii).

On the axis OY we have in absolute value

$$
\mathrm{OC}=\mathrm{OC}^{\prime}=h / 4 f
$$

On the parallel to $O Y$ at the distance $\mathrm{OF}=f$ we have

$$
\mathrm{FA}=4 \mathrm{FF}^{\prime}=h / 2 f \text {, }
$$

and on the parallel

$$
x=-f, \mathrm{~F}_{1} \mathrm{~F}_{1}^{\prime}=\mathrm{FF}^{\prime}=h / 8 f \text {. }
$$

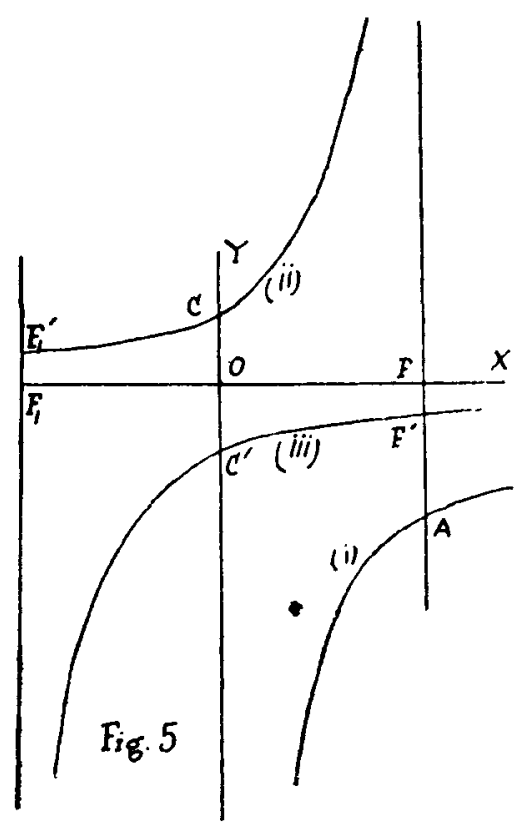


Having drawn these three curves, we have for $y / \sqrt{ }\left(f^{2}-x^{2}\right)$ the ratio of the ordinate of the curve to the ordinate of the circle, the value

$$
\frac{y}{\sqrt{ }\left(f^{2}-x^{2}\right)}=1+z_{1}+z_{2}+z_{3},
$$

each $z$ carrying its own particular sign.

Thus the ordinate of the locus of $\mathrm{D}$ is expressible as an algebraic function of the ordinates of five simple curves, the circle $x^{2}+y^{2}=f^{2}$, the line $z=1$, and the three equilateral hyperbolae (i), (ii), and (iii).

\section{Chapter II.}

Solution of the principal problem

The co-ordinates of the point $\mathrm{D}$ are

$$
x=f \sin \phi, y=f \cos \phi-h \cot 2 \phi .
$$

To obtain the value of $\phi$ we express the fact that the three points A, D, B are collinear.

The co-ordinates of $\mathrm{A}$ are $x=h, y=0$; those of $\mathrm{B}$ are $x=0$, $y=\mathrm{BI}^{\prime}-\mathrm{OI}^{\prime}=\frac{h}{2}(\cot \mu-\cot 2 \phi)$.

If we call $\xi$ and $\eta$ the coordinates of any point on $\mathrm{AB}$, we have

$$
\frac{\xi}{h}+\frac{\eta}{\frac{h}{2}(\cot \mu-\cot 2 \phi)}=1
$$

as the equation of this line, an equation which must be satisfied when we put $x$ for $\xi$ and $y$ for $\eta$, i.e., the coordinates of D.

We thus get

$$
\frac{f}{h} \sin \phi+\frac{f \cos \phi-h \cot 2 \phi}{\frac{h}{2}(\cot \mu-\cot 2 \phi)}=1
$$

an equation in which everything is known except the angle $\phi$.

Solving for $\frac{f}{h}$ we have

$$
\frac{f}{h}[\sin \phi(\cot \mu-\cot 2 \phi)+2 \cos \phi]=\cot \mu+\cot 2 \phi,
$$


and on using the identity

$$
\cot \mu \pm \cot 2 \phi=\frac{\sin (2 \phi \pm \mu)}{\sin \mu \sin 2 \phi}
$$

we obtain

$$
\frac{f}{h}=\frac{\sin (2 \phi+\mu)}{\sin \phi \sin (2 \phi-\mu)+2 \cos \phi \sin 2 \phi \sin \mu},
$$

which reduces to

$$
\frac{f}{h}=\frac{\sin (2 \phi+\mu)}{\sin \mu \sin \phi+\sin 2 \phi \sin (\phi+\mu)}
$$

an equation which only contains the sines of angles occurring in the figures of the triangles CGI, CGB in Figure 1, where G is the intersection of $C D$ and $B I$.

Thus

$$
\mu=\mathrm{CBI}, \phi=\mathrm{BCG}=\mathrm{GCI}, 2 \phi=\mathrm{BCA}, 2 \phi+\mu=\mathrm{GIA}, \phi+\mu=\mathrm{CGI} \text {. }
$$

As a verification of our formulae, we can apply them to an equilateral' triangle. In this case $f=m=h$, and we can equate them all to unity. Also $\mu=\phi$.

Thus $\sin \phi=\sin \frac{\pi}{6}=\frac{1}{2} ; \cos \phi=\frac{\sqrt{ } 3}{2} ; \sin 2 \phi=\sin \frac{\pi}{3}=\frac{\sqrt{ } 3}{2} ;$

$$
\cos 2 \phi=\frac{1}{2} ; \cot 2 \phi=\frac{1}{\sqrt{ } 3} ; \cot \mu=\cot \sqrt{ } 3 .
$$

Equation (6) then becomes

$$
\frac{1}{2}+\frac{\frac{\sqrt{ } 3}{2}-\frac{1}{\sqrt{ } 3}}{\frac{1}{2}\left(\sqrt{ } 3-\frac{1}{\sqrt{ } 3}\right)}=\frac{1}{2}+\frac{1}{2}=1 .
$$

If we use equation $(7)^{\prime}$ we have $\sin (2 \phi+\mu)-\sin 3 \phi=\sin \frac{\pi}{2}=1$, $\sin \mu \sin \phi=\sin ^{2} \phi=\frac{1}{4}, \sin 2 \phi \sin (\phi+\mu)=\sin \frac{\pi}{3} \sin \frac{\pi}{3}=\frac{3}{4}$, and finally $\frac{f}{h}=\frac{1}{f+\frac{3}{4}}=1$, which gives $f=h$.

We could take as abscissae the values of $\sin \phi$, which vary from 0 to 1 , and construct the curve $z=F(\sin \phi)$, where we call $z$ the 
ordinate, the value of the right side of equation (7). Having traced this curve, we only need to cut it by the straight line $z=f / h$ to determine the value of $\sin \phi$, which satisfies (7).

To adapt (7) to logarithmic calculation, write it in the form

$$
\frac{f}{h}=\frac{\frac{\sin (2 \phi+\mu)}{\sin \mu \sin \phi}}{1+\frac{2 \cos \phi \sin (\phi+\mu)}{\sin \mu}} .
$$

Denoting the second term of the denominator by $\tan ^{2} \theta$, or $-p$ according to its sign, we obtain

$$
\begin{gathered}
\frac{f}{h}=\frac{\sin (2 \phi+\mu)}{\sin \mu \sin \phi} \cos ^{2} \theta \\
\frac{f}{h}=\frac{\sin (2 \phi+\mu)}{\sin \mu \sin \phi(1+p)(1-p)} .
\end{gathered}
$$

\section{Discrssion of the formulae}

The limits of $\phi$, half the angle of a triangle, are 0 and $\frac{\pi}{2}$.

The triangle disappears in the extreme cases. The formulae (6) and (7) show this impossibility. For $\phi=0$ the first reduces to $2=1$, the second to $\frac{f}{h}=\infty$. For $\phi=\frac{\pi}{2}$ we have from (6) by making $\cot 2 \phi=-\infty, f / h+2=1$ which is impossible, and (7) reduces to $f / h=-\frac{\sin \mu}{\sin \mu}=-1$, the same result.

The equation (7) further limits the possible values of $\phi$.

We have $f / h=0$ for $2 \phi+\mu=\pi$, and then either $f=0$ or $h=\infty$. If $2 \phi+\mu=\pi$, the two lines $\mathrm{BI}, \mathrm{CA}$ are parallel and $\mathrm{BI}$ is indefinitely elongated. In reality $\phi$ lies between 0 and $\frac{\pi-\mu}{2}$.

\section{Particular Case. Right angled triangle.}

First case.-Suppose $\mathrm{ABC}$ is right-angled at $\mathrm{B}$ which we make coincident with $O$ (Fig. 6). Then $\mathrm{BI}=\mathrm{CI}=\mathrm{IA}$ and $\frac{f \cos \phi-h \cot 2 \phi}{\frac{h}{2}(\cot \mu-\cot 2 \phi)}$ 
which occurs in (6) takes the indeterminate form $0 / 0$. We have in fact $f \cos \phi=\mathrm{CB}=h \cot 2 \mu$ (Fig. 6) which makes the numerator vanish. Also $\cot \mu=\cot 2 \phi$ since $\mathrm{BI}=\mathrm{CI}$.
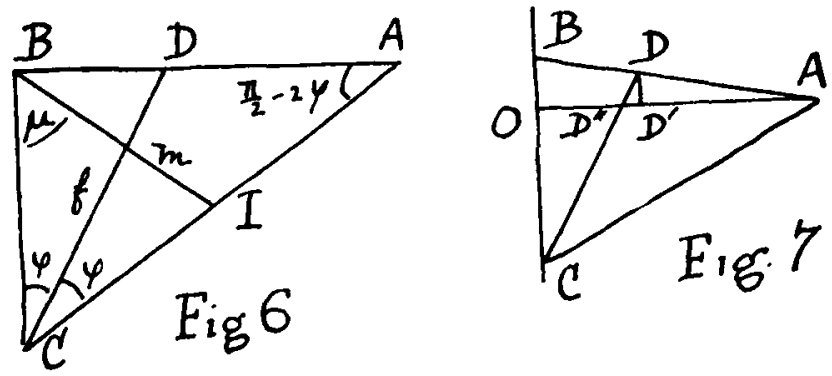

But the equation (6) still holds. The quantities

$$
\mathrm{M}=f \cos \phi-h \cot 2 \phi, \mathrm{N}=\frac{h}{2}(\cot \mu-\cot 2 \phi)
$$

are generally the ordinates of $\mathrm{D}$ and $\mathrm{B}$, and these ordinates vanish simultaneously when $\mathrm{AB}$ coincides with $\mathrm{AO}$.

We have thus the proportion $\mathrm{M} / \mathrm{N}=\mathrm{D}^{\prime} \mathrm{D} / \mathrm{OB}=\mathrm{AD}^{\prime} / \mathrm{AO}$ (Fig. 7 ), and on passing to the limit, the two points $D$ and $D^{\prime}$ coincide with $D^{\prime \prime}$ and we have, limit of $\mathrm{M} / \mathrm{N}=\mathrm{AD}^{\prime} / \mathrm{AO}$.

But $O D^{\prime}=f \sin \phi$, and therefore $(6)$ becomes

$$
\frac{f \sin \phi}{h}+\frac{\mathbf{M}}{\mathrm{N}}=\frac{\mathrm{OD}^{\prime}}{\mathrm{OA}}+\frac{\mathrm{D}^{\prime} \mathbf{A}}{\mathrm{OA}}=1
$$

The value of the biseetor is given by the equation $\mathbf{M}=0$, which gives $f=h \cot 2 \phi / \cos \phi=h \cot \mu / \cos \frac{1}{2} \mu$.

The triangle is thus completely defined by the perpendicular $h$ and the mediam $m$. The angle $2 \phi$ is equal to $\mu$.

Thesides are $\mathrm{CA}=b=2 m, \mathrm{BA}=c=h, \mathrm{BC}=a=2 m \cos \mu=\sqrt{ }\left(b^{2}-h^{2}\right)$.

The equation (7) in this particular case, gives

$$
\frac{f}{h}=\frac{\sin 4 \phi}{2 \cos \phi \sin 2 \phi \sin \mu}=\frac{\cos 2 \phi}{\cos \phi \sin \mu}=\frac{\cot \mu}{\cos \frac{\mu}{2}} .
$$


Second Case.-Triangle right angled at $\mathrm{A}$ (Fig. 8).

The point $D$ (Fig. 8 ) is equidistant from $\mathrm{CA}$ and $\mathrm{CB}$, so that $\mathrm{DA}=\mathrm{DD}^{\prime}$.

Thus $\mathrm{D}$ is on the parabola $\mathrm{LL}^{\prime}$ the envelope of the bisectors and is the point for which the tangent CD has the given length $f$.

The problem is solved by seeking this point $D$ and producing the focal radius $A D$ to meet $O Y$ in $B$.

We have

$$
\begin{gathered}
\mathrm{OC}=\mathrm{OA} \cot 2 \phi=h \cot 2 \phi, \\
\mathrm{OB}=h \tan 2 \phi . \\
\therefore a=\mathrm{OC}+\mathrm{OB}=h(\cot 2 \phi+\tan 2 \phi) \\
=2 h / \sin 4 \phi .
\end{gathered}
$$

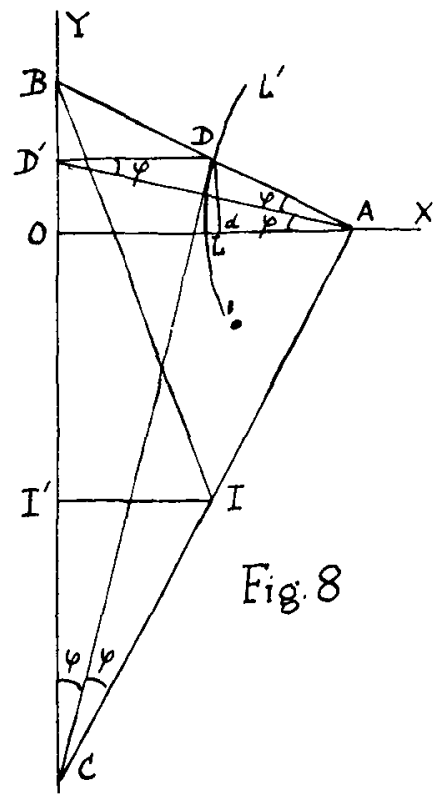

$$
\begin{aligned}
& b=\mathrm{AC}=h / \sin 2 \phi, c=\mathrm{AB}=h / \cos 2 \phi . \\
& d \mathrm{D}=\mathrm{OD}^{\prime}=\mathrm{M}=h \tan \phi, \mathrm{OB}=\mathrm{N}=h \tan 2 \phi .
\end{aligned}
$$

The equation (6) in this case becomes

or

$$
\begin{aligned}
& \frac{f}{h} \sin \phi+\frac{\tan \phi}{\tan 2 \phi}=1 \\
& \frac{f}{h} \sin \phi=\frac{1}{2 \cos ^{2} \phi} .
\end{aligned}
$$

Let $\sin \phi=u, \cos ^{2} \phi=1-u^{2}$, and we get $u-u^{3}=h / 2 f^{\circ}$ a cubic equation, which is easily soluble, for $u$ or $\sin \phi$.

$\mathrm{BI}=m$ is determined by the construction, or can be found by means of the equation

$$
\mathrm{N}=\frac{h}{2}(\cot \mu-\cot 2 \phi) .
$$

This gives

$$
\cot \mu=\cot 2 \phi+2 \mathrm{~N} / h=\cot 2 \phi+2 \tan 2 \phi=2\left(1+\sin ^{2} 2 \phi\right) / \sin 4 \phi .
$$

